\title{
Optical rogue waves in parametric three-wave mixing and coherent stimulated scattering
}

\author{
Shihua Chen, ${ }^{1, *}$ Fabio Baronio, ${ }^{2}$ J. M. Soto-Crespo, ${ }^{3}$ Philippe Grelu,,${ }^{4,}$ Matteo Conforti, ${ }^{5}$ and Stefan Wabnitz ${ }^{2}$ \\ ${ }^{1}$ Department of Physics, Southeast University, Nanjing 211189, China \\ ${ }^{2}$ Dipartimento di Ingegneria dell'Informazione, Università di Brescia, Via Branze 38, 25123 Brescia, Italy \\ ${ }^{3}$ Instituto de Óptica, Consejo Superior de Investigaciones Científicas (CSIC), Serrano 121, Madrid 28006, Spain \\ ${ }^{4}$ Laboratoire ICB, U.M.R. 6303 C.N.R.S., Univ. Bourgogne Franche-Comté, 9 avenue A. Savary, F-21078 Dijon, France \\ ${ }^{5}$ PhLAM/IRCICA UMR 8523/USR 3380, CNRS-Université Lille 1, F-59655 Villeneuve d'Ascq, France
}

(Received 8 July 2015; published 24 September 2015)

\begin{abstract}
We provide a systematic study of the rogue-wave dynamics in phase-matched weakly dispersive nonlinear media, which arise either from the parametric mixing or from the stimulated backscattering process, both of which are modeled by the three-wave resonant interaction equations. The explicit rogue-wave solutions up to the second order are presented. These solutions reveal the markedly different vector rogue-wave behaviors of the parametric and the scattering processes. In the degenerate situation where two interacting optical fields share the same group velocity, the sum of the intensities of this pair of optical wave components is spatiotemporally invariant. The close link between the existence of rogue-wave solutions and the presence of baseband modulation instability is confirmed. Finally, numerical simulations confirm that the generation of vector rogue waves is robust in the presence of spontaneous modulation instability activated by quantum noise.
\end{abstract}

DOI: 10.1103/PhysRevA.92.033847

PACS number(s): 42.65.Tg, 05.45.Yv, 47.54.-r, 47.20.Ky

\section{INTRODUCTION}

Three-wave resonant interaction (TWRI) enjoys a prominent status in nonlinear science (e.g., plasma physics, optics, fluid dynamics, and acoustics) [1-3]. In the optical context, TWRI describes different processes such as parametric amplification, frequency conversion, transient stimulated Raman scattering (SRS) and backward or forward stimulated Brillouin scattering (SBS). As such, TWRI provides the basis for our understanding of diverse pattern-forming systems [1,4-8]. Other important domains of application of TWRI in nonlinear optics are group-velocity pulse control [9-11], ultrashort pulse train generation [12], laser-plasma interaction [13], and so on.

In the early 1970 s, the integrability of the governing equations was established, and soliton solutions were identified (see Ref. [1], and references therein). These solitons are coherent localized structures that result from a dynamic balance between the energy exchanges due to the nonlinear interaction and the convection due to the group-velocity mismatch [14]. This is in contrast to the case of quadratic solitons, where the energy flow among the waves is counterbalanced by groupvelocity dispersion (GVD) (or diffraction) [15]. Interestingly, TWRI solitons propagate with a common (or locked) velocity, despite the fact that the three waves travel with different linear group velocities before the reciprocal trapping $[9,10,16]$. This property makes such solitons very alluring in applications, since the walk-off caused by group-velocity mismatch, which usually limits the parametric frequency conversion efficiency, can be circumvented by nonlinear coupling. Moreover, when two optical waves are coupled to an acoustic wave via the SBS process, TWRI solitons may permit light to considerably slow down [10].

TWRIs can be conveniently classified according to the signs of the nonlinear coupling coefficients, as well as the

\footnotetext{
*cshua@seu.edu.cn

†philippe.grelu@u-bourgogne.fr
}

ordering of the linear group velocities of each component wave. As discussed in Ref. [1], depending on these parameters, TWRIs may feature either soliton exchange (SE) dynamics (usually termed parametric three-wave mixing in the context of nonlinear optics) or stimulated backscattering (SB). TWRI may also exhibit an explosive behavior in that the coupled waves may develop a singularity —or collapse-in a finite time. Obviously, interactions of different types display very different behaviors. For instance, in the SE situation, velocitylocked solitons possess bright structures [16], whereas in the SB situation their dark counterparts would appear [17].

Quite interestingly, it has been recently pointed out that, besides velocity-locked traveling solitons, TWRI equations also admit families of spatiotemporally localized solutions-also known as rogue waves (RWs). In Ref. [18], fundamental RW solutions were presented for SE-type interactions, modeling the sudden appearance of amplitude peaks in a basic multicomponent nonlinear wave system, whose dynamics cannot be apprehended within the more familiar scalar nonlinear Schrödinger (NLS) equation framework. Recently, the intriguing dynamics of "watch-hand-like super-rogue waves" were demonstrated to occur in such a resonant three-wave mixing process [19]. These super-RWs exhibit a relative robustness even in the presence of white-noise-like perturbations, as well as a nonoverlapping distribution property, hence facilitating their forthcoming experimental observation and diagnostics.

From the fundamental point of view, RWs are deterministic since they obey partial-differential equations, and their appearance results from the evolution of a given dynamical system from well-defined initial conditions [20]. However, in a natural environment such as the ocean, as well as in most experimental optical systems [21-25], the manifestation of RWs remains inherently unpredictable, up to the point that RWs are deemed to appear from nowhere and disappear without a trace [26-28]. This peculiar feature comes from a large sensitivity of RW evolution with respect to their initial excitation conditions. Such sensitivity is typically exacerbated in any realistic environment, where RWs can be triggered from 
noise among chaotic wave fields. The apparent unexpectedness of RWs can also be approached mathematically from their rational function expression, which is localized in both space and time. Nevertheless, the slow decay of the tails of a RW means that it remains of significant magnitude at large distances from its peak (or bottom), thus effectively sensing a major portion of the spatiotemporal domain. In support of this understanding, recent theoretical works have underlined the close relationship between RWs and baseband modulation instability (MI), namely, MI whose bandwidth comprises components of arbitrarily low frequency [29]. To understand the complexity of RW manifestations in a natural environment with random initial conditions, high-order RWs should be considered too. Indeed, RWs can be mathematically expressed by a hierarchy of rational functions that remain localized both in space and in time.

Echoing the multidisciplinary diffusion of soliton concepts a few decades ago, RW investigation is now flourishing in many fields of science, for instance, in hydrodynamics [30,31], capillary waves [32], plasma physics [33], nonlinear optics [21-24,34], and Bose-Einstein condensation [35]. It has been recently extended from scalar systems [36-38] to coupled vector (or multicomponent) wave systems [29,39-46], hence providing additional new perspectives on the rogue-wave nature.

In this work, we provide a systematic study of TWRI RWs that arise from either the SE or the SB process. Explicit RW solutions up to the second order are derived, suggesting that markedly different RW behaviors are actually allowed by the different types of mixing processes. RW dynamics in the degenerate situation of equal-velocity waves is also investigated. The link between the existence of RW solutions and the presence of baseband MI is once again reasserted. Besides, we provide numerical evidences for the robustness of the generation of the considered RW patterns in spite of the presence of a background MI.

\section{EXACT ROGUE-WAVE SOLUTIONS}

The TWRI equation that governs the propagation of three coupled waves, perfectly phase matched in a weakly dispersive nonlinear medium, can be written in dimensionless form $[1,16,18]$,

$$
\begin{aligned}
& u_{1 t}+V_{1} u_{1 x}=u_{2}^{*} u_{3}^{*}, \quad u_{2 t}+V_{2} u_{2 x}=-u_{1}^{*} u_{3}^{*}, \\
& u_{3 t}+V_{3} u_{3 x}=u_{1}^{*} u_{2}^{*},
\end{aligned}
$$

where $u_{n}(x, t)(n=1,2,3)$ are the slowly varying complex envelopes of the three fields. From a physical standpoint, these fields may denote pump, signal, and idler optical waves in the parametric mixing process that occurs in a quadratic medium $[1,4,5]$, or describe the SBS (SRS) process where the optical pump wave scatters off a material acoustic (optical) phonon wave to form the Stokes wave $[2,6,7]$. We denote the time and space variables by $t$ and $x$. As in Ref. [18], time $t$ here is assumed to be the evolution variable. Subscripts $x$ ant $t$ stand for partial derivatives, and the asterisk denotes the complex conjugation. The coefficients $V_{n}$ correspond to the relative group velocities of the three waves and we suppose $V_{1,2}>V_{3}$. Without loss of generality, we set $V_{3}=0$, which implies that Eqs. (1) are written in a reference frame comoving with $u_{3}$.

The above choice of signs before the quadratic terms is indicative of the nonexplosive character of the interaction [1]. Basically, as $V_{1}>V_{2}$, the interaction features the SE property. On the other hand, the condition $V_{1}<V_{2}$ corresponds to the SB process. We will show that in either situation, Eqs. (1) admit exact RW solutions because of their complete integrability and the nonexplosive property.

Considering the resonant conditions for the frequencies and momenta, the initial plane-wave seeds which satisfy Eqs. (1) can be expressed as

$$
\begin{aligned}
& u_{10}(x, t)=a_{1} \exp \left[-i\left(k_{1} x-\omega_{1} t\right)\right], \\
& u_{20}(x, t)=a_{2} \exp \left[i\left(k_{2} x-\omega_{2} t\right)\right], \\
& u_{30}(x, t)=i a_{3} \exp \left[i\left(k_{1}-k_{2}\right) x-i\left(\omega_{1}-\omega_{2}\right) t\right],
\end{aligned}
$$

where

$$
\begin{gathered}
k_{1}=\frac{\omega_{1}}{V_{1}}+\frac{a_{2}^{2}}{\delta V_{1}}, \quad k_{2}=\frac{\omega_{2}}{V_{2}}+\frac{a_{1}^{2}}{\delta V_{2}}, \\
a_{3}=\frac{a_{1} a_{2}}{\delta},
\end{gathered}
$$

with $a_{n}(>0)$ being the respective background heights. For convenience, we use $A=\Gamma_{1} a_{1}^{2}+\Gamma_{2} a_{2}^{2}, B=\Gamma_{1} a_{1}^{2}-\Gamma_{2} a_{2}^{2}, \Gamma_{j}=$ $V_{j} /\left(V_{1}-V_{2}\right)(j=1,2), \kappa=\omega_{1}+\omega_{2}$, and $\delta=\omega_{1}-\omega_{2}$. We note that these coupled background fields are intrinsically unstable [17], and as a result, a tiny localized deformation may lead to the generation of RWs.

By exploiting the standard Darboux transformation procedure $[42,43]$, we obtain the fundamental (first-order) RW solutions of Eqs. (1),

$$
\begin{aligned}
& u_{1}^{[1]}=u_{10}\left[1+\frac{3 \Lambda \xi \theta_{1}^{*} / \alpha_{1}^{*}}{|\xi|^{2}+\Gamma_{1} a_{1}^{2}\left|\theta_{1} / \alpha_{1}\right|^{2}+\Gamma_{2} a_{2}^{2}\left|\theta_{2} / \alpha_{2}\right|^{2}}\right], \\
& u_{2}^{[1]}=u_{20}\left[1-\frac{3 \Lambda \xi^{*} \theta_{2} / \alpha_{2}}{|\xi|^{2}+\Gamma_{1} a_{1}^{2}\left|\theta_{1} / \alpha_{1}\right|^{2}+\Gamma_{2} a_{2}^{2}\left|\theta_{2} / \alpha_{2}\right|^{2}}\right], \\
& u_{3}^{[1]}=u_{30}\left[1+\frac{3 \delta \Lambda \theta_{1} \theta_{2}^{*} /\left(\alpha_{1} \alpha_{2}^{*}\right)}{|\xi|^{2}+\Gamma_{1} a_{1}^{2}\left|\theta_{1} / \alpha_{1}\right|^{2}+\Gamma_{2} a_{2}^{2}\left|\theta_{2} / \alpha_{2}\right|^{2}}\right],
\end{aligned}
$$

where $\Lambda=\lambda_{0}-\lambda_{0}^{*} \equiv 2 i \operatorname{Im}\left(\lambda_{0}\right), \xi=t+\beta_{2} x, \alpha_{j}=\mu_{0}+$ $\lambda_{0}-(-1)^{j} \delta / 2$, and $\theta_{j}=\xi-i / \alpha_{j}(j=1,2)$. Here and for later use, we define the parameters $\beta_{n}$ as $\beta_{n}=\frac{1}{V_{1}-V_{2}}\left(\frac{a_{1}^{2}}{\alpha_{1}^{n}}+\frac{a_{2}^{2}}{\alpha_{2}^{n}}\right)$ $(n=2,3,4)$.

The specific spectral parameter $\lambda_{0}$ in Eqs. (5) is the complex root (with a nonzero imaginary part) of the discriminant condition

$$
\Delta=\sigma^{3}-\rho^{2}=0,
$$

under which the cubic equation

$$
\mu_{0}^{3}-3 \sigma \mu_{0}+2 \rho=0
$$

will have a double root $\mu_{0}$. Here

$$
\begin{gathered}
\sigma=\lambda_{0}^{2}+\frac{\delta^{2}}{12}+\frac{A}{3} \\
\rho=\frac{1}{4}\left(\delta^{2}-2 A\right) \lambda_{0}-\lambda_{0}^{3}+\frac{\delta B}{4} .
\end{gathered}
$$


Let us point out that Eqs. (5) apply to either the SE process or the SB process. Generally, one can solve Eq. (6) analytically for $\lambda_{0}$, and then solve exactly the cubic equation (7) for $\mu_{0}$, even with $B \neq 0$, although in most cases these formulas are quite lengthy. Naturally, substitution of the values of $\lambda_{0}$ and $\mu_{0}$ into Eqs. (5) results in the fundamental RW dynamics that we shall demonstrate in Sec. III. We find that the RW solutions for $V_{1}>V_{2}$ could exist in the whole regime $|\delta|<+\infty$, while those for $V_{1}<V_{2}$ (in this case, $A<0$ ) only exist in the limited regime where

$|\delta| \leqslant\left\{\frac{3}{2}\left(A^{2}-B^{2}\right)^{1 / 3}\left[(-A-B)^{1 / 3}+(-A+B)^{1 / 3}\right]-A\right\}^{1 / 2}$.

One can recall that the vector Manakov system with normal dispersion also entails a parametric condition similar to Eq. (10) for the existence of RWs $[29,43]$.

Once the parameters $\lambda_{0}$ and $\mu_{0}$ are known, the general second-order RW solutions can be cast in the following compact form:

$$
\begin{aligned}
& \frac{u_{1}^{[2]}}{u_{10}}=1+\frac{3 i \Lambda\left[R_{1}^{*}\left(R_{0} m_{22}-S_{0} m_{21}\right)+S_{1}^{*}\left(S_{0} m_{11}-R_{0} m_{12}\right)\right]}{a_{1}\left(m_{11} m_{22}-m_{12} m_{21}\right)}, \\
& \frac{u_{2}^{[2]}}{u_{20}}=1+\frac{3 i \Lambda\left[R_{0}^{*}\left(R_{2} m_{22}-S_{2} m_{21}\right)+S_{0}^{*}\left(S_{2} m_{11}-R_{2} m_{12}\right)\right]}{a_{2}\left(m_{11} m_{22}-m_{12} m_{21}\right)},
\end{aligned}
$$

$\frac{u_{3}^{[2]}}{u_{30}}=1+\frac{3 \Lambda\left[R_{2}^{*}\left(R_{1} m_{22}-S_{1} m_{21}\right)+S_{2}^{*}\left(S_{1} m_{11}-R_{1} m_{12}\right)\right]}{a_{3}\left(m_{11} m_{22}-m_{12} m_{21}\right)}$,

where

$$
\begin{gathered}
R_{0}=\gamma_{1}-i \gamma_{2} \phi \xi, \quad R_{j}=\frac{a_{j}}{\alpha_{j}}\left(i \gamma_{1}+\gamma_{2} \phi \theta_{j}\right) \\
S_{0}=\gamma_{1} p-i \gamma_{2} \phi(q \xi+g x)+\gamma_{3}-i \gamma_{4} \phi \xi \\
S_{j}=\frac{a_{j}}{\alpha_{j}}\left[\gamma_{1}\left(i p-\frac{\phi^{2} \theta_{j}+i \Lambda+i \varphi}{\alpha_{j}}\right)+i \gamma_{3}+\gamma_{4} \phi \theta_{j}\right. \\
\left.+\gamma_{2} \phi\left(q \theta_{j}+g x+\frac{\vartheta_{j} \alpha_{j}-i \phi^{2}}{3 \alpha_{j}^{3}}\right)\right] \\
m_{11}=\left|R_{0}\right|^{2}+\Gamma_{1}\left|R_{1}\right|^{2}+\Gamma_{2}\left|R_{2}\right|^{2} \\
m_{12}=R_{0}^{*} S_{0}+\Gamma_{1} R_{1}^{*} S_{1}+\Gamma_{2} R_{2}^{*} S_{2}-m_{11} \equiv m_{21}^{*} \\
m_{22}=\left|S_{0}\right|^{2}+\Gamma_{1}\left|S_{1}\right|^{2}+\Gamma_{2}\left|S_{2}\right|^{2}-m_{12}-m_{21}
\end{gathered}
$$

with $\quad \vartheta_{j}=\left(\theta_{j} \phi^{2}+3 i \Lambda+3 i \varphi\right)\left(2+i \alpha_{j} \xi\right), \quad p=-\frac{\phi^{2} \xi^{2}}{2}-$ $i \varphi \xi-i\left(\Lambda \beta_{2}-\beta_{3} \phi^{2}\right) x, \quad q=p+\frac{1}{3} \phi^{2} \xi^{2}+\frac{\Lambda-2 \varphi}{6 \mu_{0}}+\frac{(\Lambda+\varphi)^{2}}{2 \phi^{2}}$, and $\gamma_{s}(s=1,2,3,4)$ being four arbitrary complex constants (termed structural parameters). The other parameters $\phi, \varphi$, and $g$ in Eqs. (12)-(14) are defined by

$$
\begin{gathered}
\phi=\left[\Lambda\left(\alpha_{1}+\alpha_{2}-\frac{A+\delta^{2}}{3 \mu_{0}}\right)\right]^{1 / 2}, \\
\varphi=\frac{6 \Lambda \lambda_{0}-\phi^{2}}{6 \mu_{0}}, \\
g=-2 \beta_{3}(\Lambda+\varphi)+\beta_{4} \phi^{2} .
\end{gathered}
$$

In the present paper, we will also demonstrate the RW triplet dynamics in both the SE and SB situations, which are based on the exact second-order solutions (11).

\section{DIVERSE ROGUE-WAVE DYNAMICS}

Let us first discuss the fundamental RW dynamics provided by Eqs. (5), in either the SE or the SB regime. Next we shall demonstrate the intriguing properties of RW triplets in both regimes. For clarity of the underlying physics, we confine our discussions within the simple parametric condition $B=0$, which implies

$$
\frac{a_{2}}{a_{1}}=\sqrt{V_{1} / V_{2}}
$$

Under these circumstances, it follows from Eqs. (6) and (7) that

$$
\begin{aligned}
\lambda_{0} & =\frac{i}{4} \eta+\frac{i\left(4 A+\delta^{2}\right)}{12 \eta}, \\
\mu_{0} & =\frac{i}{4} \eta-\frac{i\left(4 A+\delta^{2}\right)}{12 \eta},
\end{aligned}
$$

where the parameter $\eta$ is given by

$$
\eta= \pm\left[2 \sqrt{A\left(A-2 \delta^{2}\right)}+2 A-\delta^{2}\right]^{1 / 2} .
$$

It is easily seen that, as $V_{1}>V_{2}$ (noting that $A>0$ ), the value of $\eta$ will be real for $|\delta| \leqslant \sqrt{A / 2} \equiv \delta_{h}$, but it becomes complex when $|\delta|>\delta_{h}$. However, as $V_{1}<V_{2}$ (which means $A<0$ ), $\eta$ will always be real in the regime $|\delta| \leqslant 2 \sqrt{-A} \equiv \delta_{m}$. We can see that as $\delta>\delta_{m}$, the RW solutions will be forbidden in the latter case, according to the parameter condition (10). Besides, we note that both solutions (5) and (11) involve structures which are independent of the parameter $\kappa$. For this reason, we will always assume $\kappa=0$ in our numerical examples given below.

\section{A. Fundamental rogue waves}

Therefore, by use of Eqs. (22) and (23), the fundamental RW solutions (5) can be reduced to their most explicit forms. Specifically, in the SB situation or in the SE situation with $|\delta| \leqslant \delta_{h}$, RW solutions can be expressed as

$$
\begin{aligned}
& \frac{u_{1}^{[1]}}{u_{10}}=1+\frac{[i \delta(t-\chi x)-i \varrho x-1] \ell+4 i v \delta^{2} x}{\delta^{2}\left[(t-\chi x)^{2}+\epsilon^{2} v^{2} x^{2}+1 / \eta^{2}\right]}, \\
& \frac{u_{2}^{[1]}}{u_{20}}=1+\frac{[i \delta(t-\chi x)+i \varrho x-1] \ell-4 i v \delta^{2} x}{\delta^{2}\left[(t-\chi x)^{2}+\epsilon^{2} v^{2} x^{2}+1 / \eta^{2}\right]}, \\
& \frac{u_{3}^{[1]}}{u_{30}}=1-\frac{2 i \delta(t-\chi x) \ell+2\left(\ell-\delta^{2} / A\right)}{\delta^{2}\left[(t-\chi x)^{2}+\epsilon^{2} v^{2} x^{2}+1 / \eta^{2}\right]},
\end{aligned}
$$

where $\epsilon=\eta-\frac{4 A+\delta^{2}}{\eta}, \chi=\frac{V_{1}+V_{2}}{2 V_{1} V_{2}}, v=\frac{V_{1}-V_{2}}{4 \delta V_{1} V_{2}}, \varrho=2(2 A+$ $\left.\delta^{2}\right) \nu$, and

$$
\ell= \begin{cases}1+\sqrt{1-2 \delta^{2} / A}, & \text { SB, } \\ 1-\sqrt{1-2 \delta^{2} / A}, & \text { SE and }|\delta| \leqslant \delta_{h} .\end{cases}
$$


Otherwise, for the SE case with a large frequency difference $|\delta| \geqslant \delta_{h}$, our solutions take the form

$$
\begin{aligned}
& \frac{u_{1}^{[1]}}{u_{10}}=1+\frac{i e_{2}[\tau-2(m+\delta)(\iota+1) v x]-e_{1} \iota \delta / A}{\delta^{2} \tau^{2}+4 h^{2}\left(\delta^{2}-m^{2}\right) v^{2} x^{2}+\delta^{2} / h^{2}}, \\
& \frac{u_{2}^{[1]}}{u_{20}}=1+\frac{i e_{1}[\tau-2(m-\delta)(\iota+1) v x]-e_{2} \iota \delta / A}{\delta^{2} \tau^{2}+4 h^{2}\left(\delta^{2}-m^{2}\right) v^{2} x^{2}+\delta^{2} / h^{2}}, \\
& \frac{u_{3}^{[1]}}{u_{30}}=1-\frac{2 i \delta[\tau-2 m v x(\iota-1 / \iota)]+2 \delta^{2} / A}{\delta^{2} \tau^{2}+4 h^{2}\left(\delta^{2}-m^{2}\right) v^{2} x^{2}+\delta^{2} / h^{2}},
\end{aligned}
$$

where $\tau=t-(\chi-2 \iota m v) x, e_{1}=\delta+m / \iota, e_{2}=\delta-m / \iota$, $\iota=1 / 2+\sqrt{1 / 4+A / \delta^{2}}$, and $h=\sqrt{A(1+1 / \iota)}$ and $m=$ $\pm \sqrt{l \delta^{2}-A}$ are the real and imaginary parts of $\eta$, respectively. The plus-minus sign in $m$ means that there are two possible RW structures which could coexist for the same given initial plane-wave parameters [41]. In the following, we will take the positive sign for $m$, unless otherwise stated.

We need to emphasize that the above analytic solutions (25) and (27) have been translated along the $t$ axis, so that their center is exactly on the origin. Besides, these solutions have been expressed as a ratio of second-order polynomials, with the real and imaginary parts being clearly separated. This series of operations may help to understand the wave characteristics in a straightforward manner. For instance, one can find directly from Eqs. (25) that the relative amplitudes of three RW components at the origin are given by

$$
E_{1}=E_{2}=\left|1-\frac{\eta^{2} \ell}{\delta^{2}}\right|, \quad E_{3}=\left|1-\frac{2 \eta^{2} \ell}{\delta^{2}}+\frac{2 \eta^{2}}{A}\right|,
$$

where we define $E_{n}=\frac{1}{a_{n}}\left|u_{n}^{[1]}(0,0)\right|(n=1,2,3)$. In the same way, we obtain from Eqs. (27) directly another set of relative central amplitudes

$$
E_{j}=\left|1-\frac{e_{j} l h^{2}}{\delta A}\right|(j=1,2), \quad E_{3}=\left|1-\frac{2 h^{2}}{A}\right| .
$$

Obviously, the central amplitudes given by Eqs. (28) and (29) depend on the frequency difference $\delta$. For illustration, we display in Fig. 1 the dependence of the relative central amplitude $E_{n}$ on $\delta$, in the SB situation [see panel (a)] and in the SE situation [see panel (b)], respectively. For the former situation, we used a set of parameters $a_{1}=2, V_{1}=1$, and $V_{2}=4$, which means $A=-8 / 3$ and $B=0$. For the latter situation, we used another set of parameters $a_{1}=1, V_{1}=4$, and $V_{2}=1$ so that $A=8 / 3$ and $B=0$. Note that the values of $a_{2}$ and $a_{3}$ are determined by Eqs. (4) and (21). For the sake of brevity, here and in what follows, unless otherwise stated, we only take the positive domain $\delta>0$ (i.e., $\omega_{1}>\omega_{2}$ ) into account. The problem in the negative domain $\delta<0$ can be treated along similar lines. As can be seen from Fig. 1(a), in the SB situation the RW components $u_{1}$ and $u_{2}$ have a vanishing central amplitude at $\delta=\delta_{a}$, and on the other hand, the third wave amplitude $u_{3}$ vanishes at $\delta=\delta_{b}$, where

$$
\delta_{a}=\frac{1}{2} \sqrt{-6 A}, \quad \delta_{b}=\frac{1+\sqrt{3}}{2} \sqrt{-3^{1 / 2} A} .
$$

Also, in the SE case, we find from Fig. 1(b) that the components $u_{2}$ and $u_{3}$ have a central amplitude vanishing at $\delta=\delta_{d}$ and $\delta_{c}$,
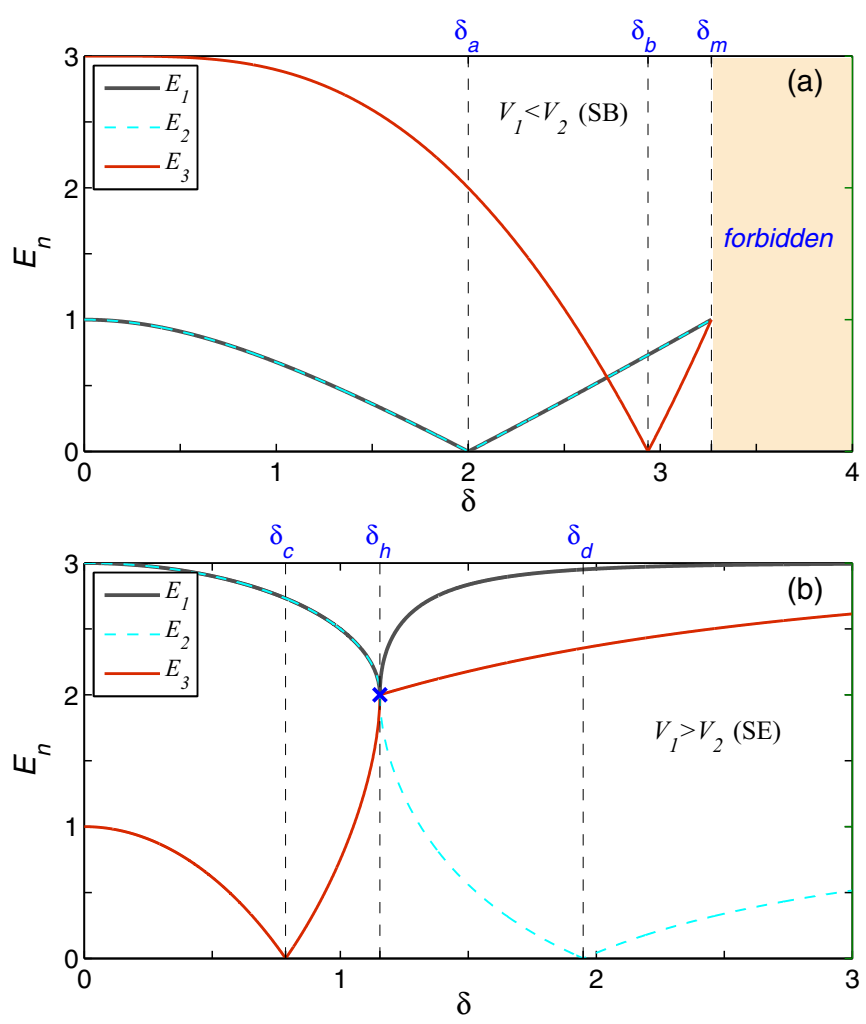

FIG. 1. (Color online) Dependence of the relative central amplitudes $E_{n}$ on $\delta$, for either the (a) SB or (b) SE process. In (a), the background parameters are $a_{1}=2, V_{1}=1$, and $V_{2}=4$, while in (b) they are specified by $a_{1}=1, V_{1}=4$, and $V_{2}=1$.

respectively, where

$$
\begin{aligned}
& \delta_{c}=\frac{-1+\sqrt{3}}{2} \sqrt{3^{1 / 2} A}, \\
& \delta_{d}=\sqrt{\left[(\sqrt{2}+1)^{4 / 3}+(\sqrt{2}-1)^{4 / 3}+5\right] A / 6} .
\end{aligned}
$$

It is worth noting that, if we chose a minus sign for $m$, then it will be the field component $u_{1}$, rather than $u_{2}$, that becomes zero at $\delta=\delta_{d}$. In addition, Fig. 1(a) shows further that the RW states are totally forbidden to occur in the SB process as soon as $\delta>\delta_{m}$.

As a matter of fact, different types of fundamental RW structures do appear as $\delta$ is varied, because the latter is closely related to the values of the central amplitude of each field component. As in previous works, we may define a RW to be bright (Peregrine-type [36]) whenever its peak amplitude at the center is more than twice the background height $[30,39]$. Naturally, we refer to their cousins-RWs with one single hole on a nonzero background - as being dark [40,43]. Of particular interest is the black RW, which corresponds to a special dark RW whose intensity exactly falls to zero at the dip center. For convenience of our subsequent discussion, we may also loosely identify RW states with a double-peak-double-dip structure as intermediate waves.

In the frame of the above phenomenological descriptions, we find that in the SB case [see Fig. 1(a)] the three RW components $u_{n}$ exhibit intermediate-intermediate-bright (IIB) structures as $\delta<\delta_{a}$, dark-dark-intermediate (DDI) structures 

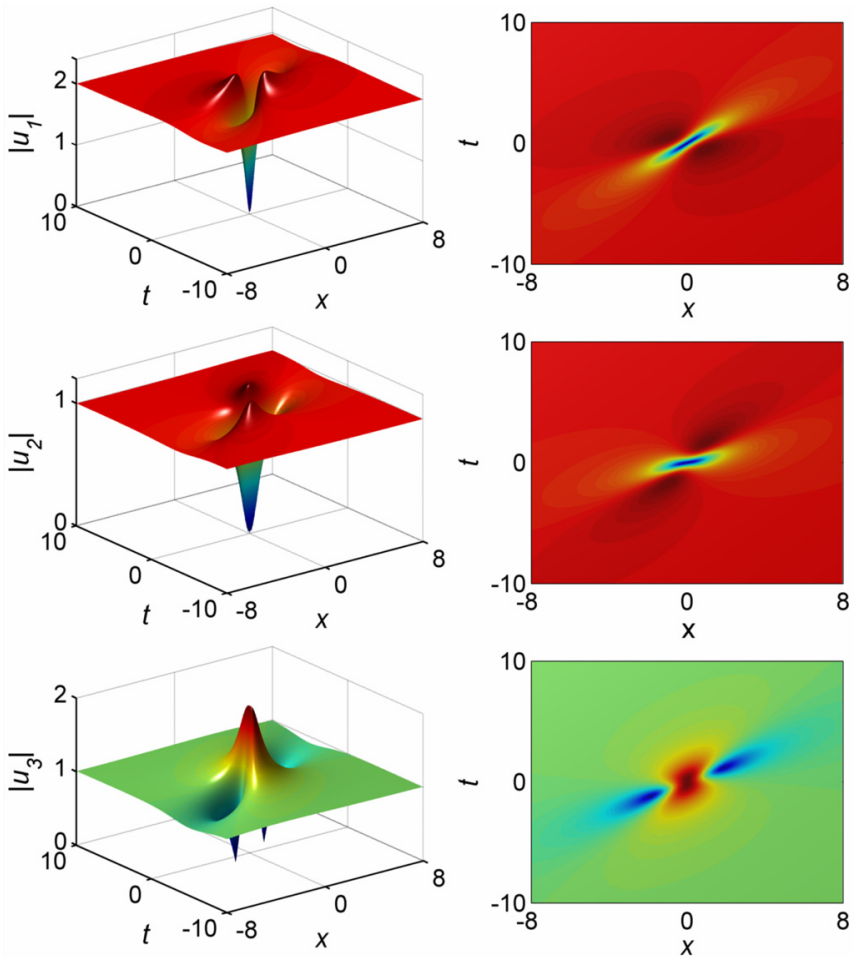

FIG. 2. (Color online) DDB RW states occurring in the SB process for $\delta=\delta_{a}$. The parameters are given by $a_{1}=2, V_{1}=1$, and $V_{2}=4$, the same as in Fig. 1(a). The left column shows the surface plots, while the right column shows the corresponding contour distributions.

as $\delta_{a}<\delta<\delta_{b}$, and finally dark-dark-dark (DDD) structures as $\delta_{b} \leqslant \delta<\delta_{m}$. Particularly, at $\delta=\delta_{a}$, dark-dark-bright (DDB) RW structures appear, as illustrated in Fig. 2, using otherwise identical background parameters as in Fig. 1(a). Obviously, the first two field components exhibit a black structure, whereas the third component is of a bright nature, with its peak amplitude reaching just twice the background height.

On the other hand, in the SE case [see Fig. 1(b)], one finds that the three RW components are of the bright-brightdark (BBD) type as $\delta \leqslant \delta_{c}$, or $\mathrm{BDB}$ as $\delta \geqslant \delta_{d}$, but remain bright-bright-intermediate (BBI) or BIB as $\delta_{c}<\delta<\delta_{d}$. As an illustration, we demonstrate in Fig. 3 the RW states for $\delta=$ $\delta_{c}$ (left column) and for $\delta=\delta_{d}$ (right column), respectively, with the other background parameters kept unchanged. It is seen that at either $\delta=\delta_{c}$ or $\delta=\delta_{d}$, there are always two RW components which feature bright structures. However, the third field amplitude $\left|u_{3}\right|$ in the former case and the second amplitude $\left|u_{2}\right|$ in the latter case have a black wave structure. Furthermore, as one can prove, the three RW components at $\delta=\delta_{h}$, corresponding to the blue cross in Fig. 1(b), are of the bright-bright-bright (BBB) type, all with a peak amplitude equal to just twice the background height. We should point out that for $\delta=\delta_{h}$ and $B=0$, the cubic equation (7) has a triple zero root. As discussed in Ref. [18], in this special SE case the fundamental RW dynamics can be expressed in terms of a more general solution (involving additional structures such as RW doublets) than the one which is reported in Eqs. (5).
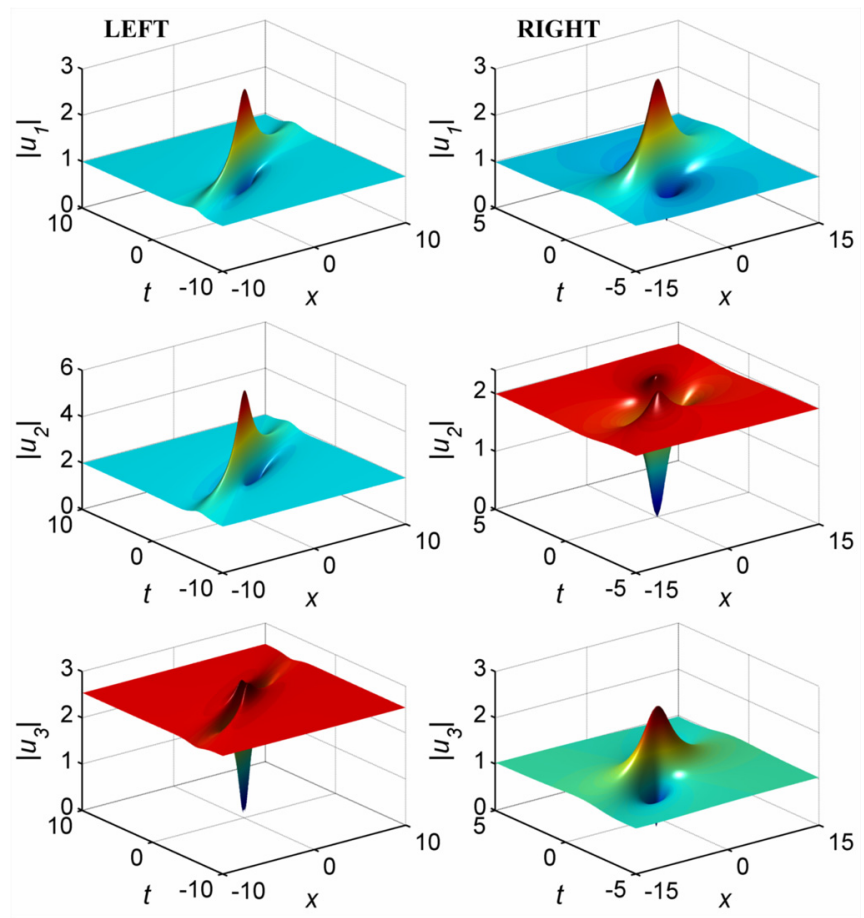

FIG. 3. (Color online) Typical (left) BBD and (right) BDB RW states occurring in the SE process, formed at $\delta=\delta_{c}$ and $\delta=\delta_{d}$, respectively. The other parameters are given by $a_{1}=1, V_{1}=4, V_{2}=$ 1, the same as in Fig. 1(b).

Therefore, we may conclude the following: in the SB situation, at least two RW components will be dark as $\delta \geqslant \delta_{a}$ (see Fig. 2), whereas in the SE case, there are at least two RW fields which are bright (see Fig. 3). The markedly different RW dynamics in the SB and SE cases are analogous to what occurs for RWs of the Manakov system when considering the defocusing and focusing situations (or equivalently the normal and anomalous GVD situations), respectively [29,42,43]. Since the behaviors shown in Figs. 1(a) and 1(b) exhibit different dynamical characteristics in the SE and SB cases, one may naturally ask whether the TWRI equations (1) admit exact RW solutions in the degenerate case $V_{2}=V_{1}$.

To this end, we merely consider the simple case $B=0$, which implies that $a_{2}=a_{1}$ [according to Eq. (21), with the assumption of $V_{2}=V_{1}$ ]. We find that the TWRI equations (1) do admit the fundamental RW solutions in this degenerate situation. These very special solutions can be obtained from Eqs. (25) by taking the limit $V_{2} \rightarrow V_{1}$ in the SB case, with the resultant form given by

$$
\begin{aligned}
& \frac{u_{1}^{[1]}}{u_{10}}=1+\frac{2 i\left(t-x / V_{1}\right) \delta-4 i a_{1}^{2} x /\left(\delta V_{1}\right)-2}{\delta^{2}\left(t-x / V_{1}\right)^{2}+4 a_{1}^{4} x^{2} /\left(\delta^{2} V_{1}^{2}\right)+1}, \\
& \frac{u_{2}^{[1]}}{u_{20}}=1+\frac{2 i\left(t-x / V_{1}\right) \delta+4 i a_{1}^{2} x /\left(\delta V_{1}\right)-2}{\delta^{2}\left(t-x / V_{1}\right)^{2}+4 a_{1}^{4} x^{2} /\left(\delta^{2} V_{1}^{2}\right)+1}, \\
& \frac{u_{3}^{[1]}}{u_{30}}=1-\frac{4 i\left(t-x / V_{1}\right) \delta+4}{\delta^{2}\left(t-x / V_{1}\right)^{2}+4 a_{1}^{4} x^{2} /\left(\delta^{2} V_{1}^{2}\right)+1} .
\end{aligned}
$$

As can be easily checked, in Eqs. (32) the three field components have relative central amplitudes equal to 1,1 , 

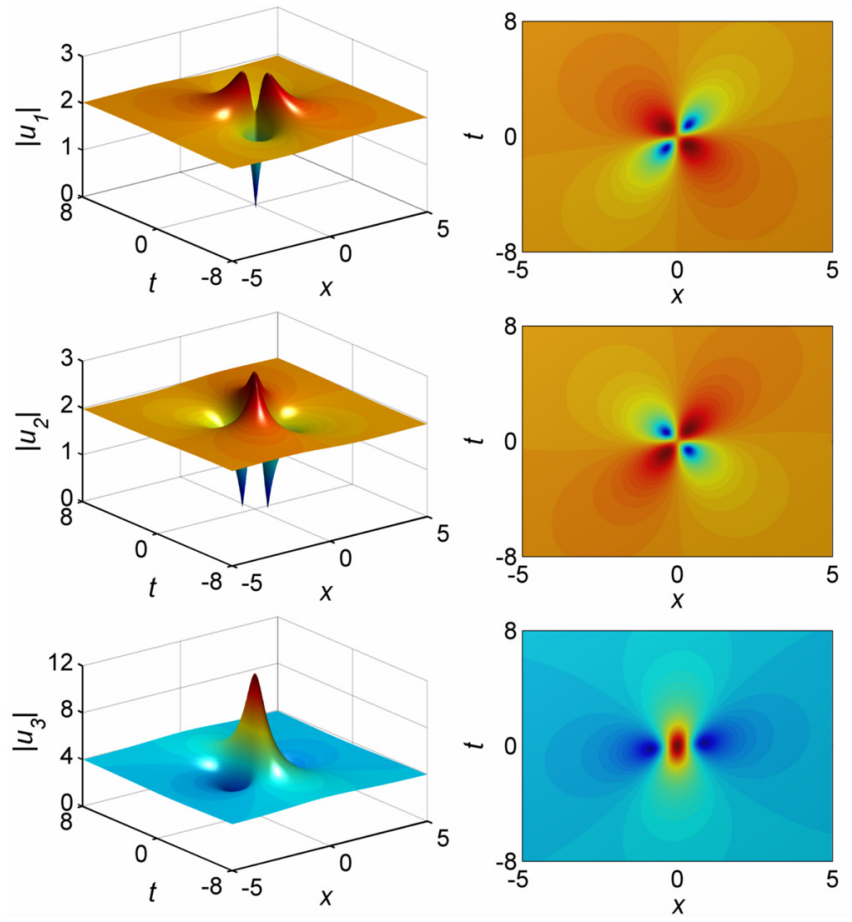

FIG. 4. (Color online) Typical IIB RW states formed at $\delta=1$ in the degenerate situation $V_{2}=V_{1}=4$. The initial background parameters are $a_{1}=2, a_{2}=2, a_{3}=4$. Left: surface plots; right: corresponding contour distributions.

and 3, respectively. According to our denominations above, they correspond to IIB structures, which are displayed in Fig. 4. Note that the first two wave components do feature a double-peak-double-dip structure around the origin: in both components, deformations to the background appear with opposite symmetry. In particular, we find that $\left|u_{1}\right|^{2}+\left|u_{2}\right|^{2}=$ $a_{1}^{2}+a_{2}^{2}$, namely, the sum of the first two wave intensities is spatiotemporally invariant. This holds true even for $B \neq 0$ (or $a_{1} \neq a_{2}$ ), in which case the three RW components can be of the BDB or DBB type. This dynamical property is completely different from what occurs in the SE or SB scenario, where this sum is not conserved. Such a property may be linked to the related behavior of the sine-Gordon equation [47], but further investigation is needed.

We should point out that the solutions (32) cannot be derived from Eqs. (25) or (27) in the SE situation, in the limit of $V_{2} \rightarrow V_{1}$. This fact further confirms that RW states in the SB and SE situations work in a distinctly different manner. On a qualitative level, we find that, when compared with the degenerate case, the nondegenerate situation favors the presence of elongated asymmetric RW structures, which culminate in the so-called watch-hand-like RWs; see, for example, Fig. 1 in Ref. [19].

\section{B. Rogue-wave triplets}

In comparison with the fundamental RWs of Sec. III A, the second-order RW solutions (11) may exhibit a great variety of complex patterns $[19,31,42-46]$. Here, for brevity, we shall exclusively address the triplet dynamics, with the assumption $B=0$. In this case, the values of $\lambda_{0}$ and $\mu_{0}$ are explicitly given
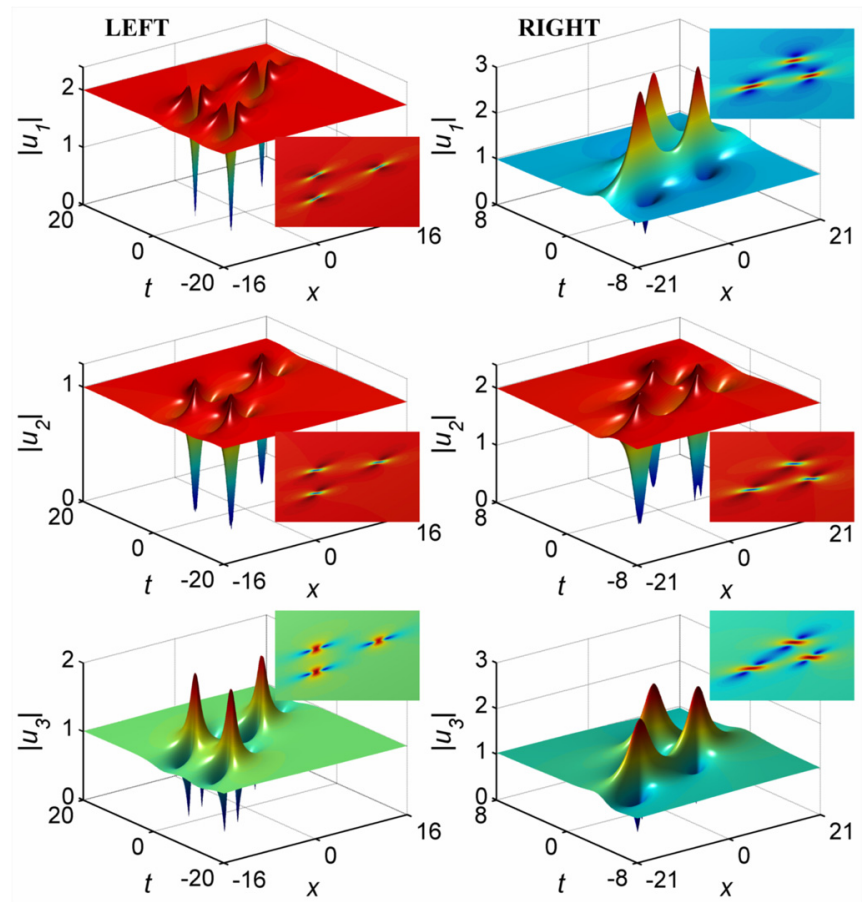

FIG. 5. (Color online) Typical (left) DDB and (right) BDB RW triplets formed at $\delta=\delta_{a}$ in the SB situation and at $\delta=\delta_{d}$ in the SE situation, respectively. We use the same background parameters as in Figs. 2 and 3 (right), and show the contour distributions by the insets. The four structural parameters are the same in both situations, given by $\gamma_{1}=5, \gamma_{2}=1$, and $\gamma_{3}=\gamma_{4}=0$.

by Eqs. (22) and (23). We show that these RW triplets will have almost the same dynamics as the corresponding fundamental RW solutions. Specifically, in the SB situation, rogue triplets also exhibit IIB, DDI (including DDB), or DDD structures in the regime $\delta<\delta_{a}, \delta_{a} \leqslant \delta<\delta_{b}$, or $\delta_{b} \leqslant \delta<\delta_{m}$, respectively. Quite differently, in the SE situation these triplets will be BBD as $\delta \leqslant \delta_{c}$ or BDB as $\delta \geqslant \delta_{d}$, but BBI or BIB (including BBB) as $\delta_{c}<\delta<\delta_{d}$.

To give some specific examples, we illustrate in Fig. 5 the DDB triplets for the SB case (left column) and the BDB triplets for the SE case (right column), by using identical background parameters as in Figs. 2 and 3 (right), respectively. In both situations, the structural parameters are taken to be the same, i.e., $\gamma_{1}=5, \gamma_{2}=1$, and $\gamma_{3}=\gamma_{4}=0$. It is clear that the three field components have the same evolution characteristics as in Figs. 2 and 3 (right), apart from each having a triplet feature in the present case. We point out that the three constituents of each triplet are usually held in a triangle position, but their layout is not fixed, and it can change with the values of the four structural parameters $\gamma_{s}$.

Interestingly, in the degenerate situation $\left(V_{2}=V_{1}\right)$ the second-order RW solutions can also be obtained from Eqs. (11) by taking the limit $V_{2} \rightarrow V_{1}$, once again by considering the SB case. Moreover, the sum of intensities $\left|u_{1}\right|^{2}+\left|u_{2}\right|^{2}$ is still maintained constant, and it remains equal to $a_{1}^{2}+a_{2}^{2}$. Figure 6 displays the IIB RW triplets which are formed for $\delta=1$, with $a_{2}=a_{1}=2$ and $V_{2}=V_{1}=4$. The structural parameters are exactly the same as in Fig. 5. 

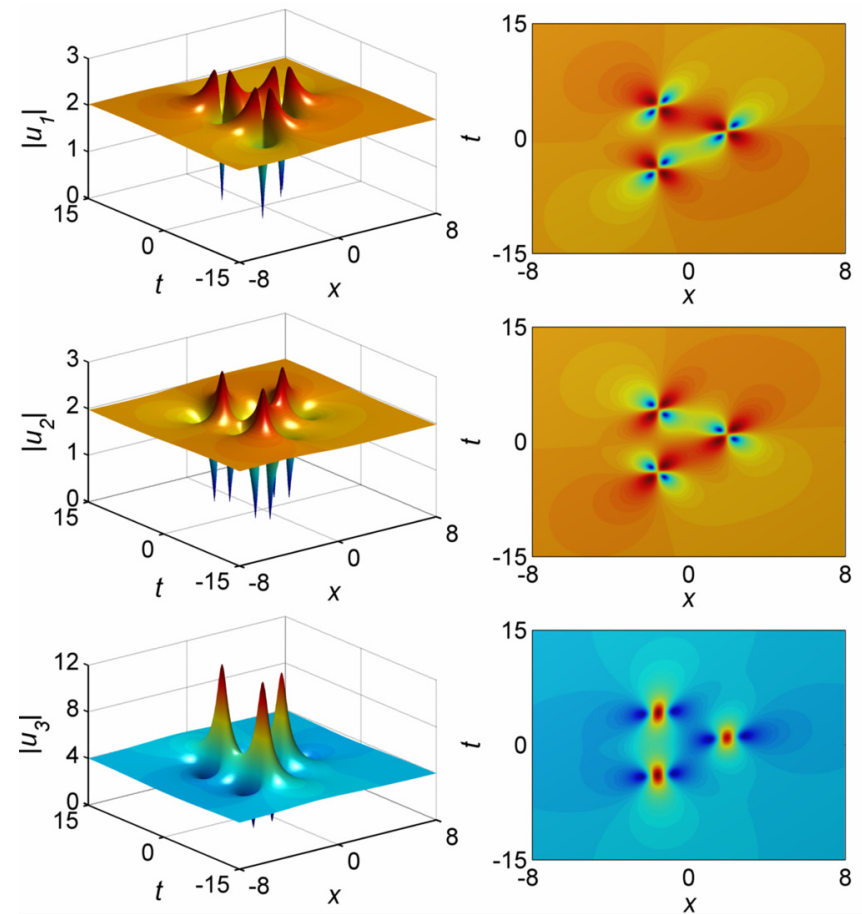

FIG. 6. (Color online) IIB RW triplets formed at $\delta=1$ for the degenerate situation. The background parameters are identically the same as in Fig. 4, while the four structural parameters are specified by $\gamma_{1}=5, \gamma_{2}=1$, and $\gamma_{3}=\gamma_{4}=0$.

\section{Energy exchange}

Let us simply comment on the energy exchanges among the three RW components $[5,16,18,19]$. We recall that Eqs. (1) represent an infinite-dimensional Hamiltonian dynamical system that obeys the conservation laws or Manley-Rowe relations

$$
\frac{d}{d t}\left(I_{1}+I_{2}\right)=\frac{d}{d t}\left(I_{2}+I_{3}\right)=0,
$$

where $I_{n}(t)=\frac{1}{2} \int_{-\infty}^{+\infty}\left(\left|u_{n}\right|^{2}-\left|u_{n 0}\right|^{2}\right) d x \quad(n=1,2,3)$ define the effective energy of the three-wave components at a certain given time. By effective energy we mean that the energy is renormalized with respect to the constant background. As examples, we plot in Fig. 7 the energy evolutions for the fundamental RWs shown in Figs. 2 and 3 (right) [see panels (a) and (c)], and for the RW triplets shown in Figs. 5 (left) and 5 (right) [see panels (b) and (d)]. One can verify that these RWs, no matter how complex their structures are and no matter which process they result from, always obey the energy relation $I_{1}=I_{3}=-I_{2}$, which in general implies that important energy exchanges occur among the three-wave components. This is naturally expected in phase-matched parametric processes, in marked contrast to the case of coupled NLS equations where energy exchanges are excluded, except when additional coupling effects, such as four-wave mixing, are present. However, as one can see from Fig. 7, the RWs in the SB situation, either of the fundamental or triple nature, involve a less drastic energy flow than in the SE situation.

On the other hand, if we define the effective pulse energy at a given spatial position as $P_{n}(x)=\frac{1}{2} \int_{-\infty}^{+\infty}\left(\left|u_{n}\right|^{2}-\left|u_{n 0}\right|^{2}\right) d t$, then, owing to symmetry, one obtains the energy-conservation
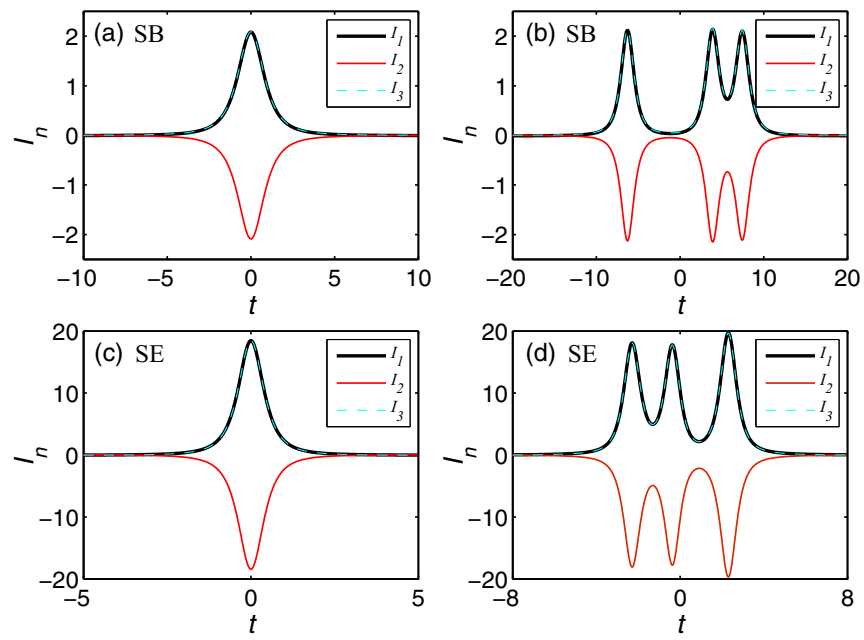

FIG. 7. (Color online) Evolutions of the effective energy $I_{n}$ with respect to $t$ for [(a), (c)] the fundamental RW structures shown in Figs. 2 and 3 (right), and [(b), (d)] the RW triplets shown in Figs. 5 (left) and 5 (right).

relations

$$
\frac{d}{d x}\left(V_{1} P_{1}+V_{2} P_{2}\right)=\frac{d}{d x}\left(V_{2} P_{2}+V_{3} P_{3}\right)=0 .
$$

It is easily concluded from Eq. (34), with $V_{3}=0$ and the boundary conditions being taken into account, that whenever the first two RW components have a zero pulse energy, i.e., $P_{1}=P_{2}=0$, still the third wave may exhibit a nonzero evolution of energy $P_{3}$ with distance. As far as this property is concerned, one can refer to Figs. 3(c) and 3(d) in Ref. [19].

\section{Rogue-wave existence and baseband MI}

The parametric condition for the RW existence can also be contrasted with the recently developed baseband-MI conjecture, which asserts that RWs can manifest only when nonzero MI gain is available for arbitrarily low modulation frequencies. This conjecture has been validated for several integrable nonlinear systems [29].

To check this, we add small-amplitude Fourier modes to the plane-wave solutions (2), and express them as $u_{n}=$ $u_{n 0}\left\{1+p_{n} \exp [-i \Omega(\mu t-x)]+q_{n}^{*} \exp \left[i \Omega\left(\mu^{*} t-x\right)\right]\right\} \quad(n=$ $1,2,3)$, where $p_{n}$ and $q_{n}$ are small amplitudes of the Fourier modes, and the parameters $\Omega$ and $\mu$ are assumed to be positive and complex, respectively $[17,19,29,40,48]$. A substitution of these perturbed plane-wave solutions into Eqs. (1) followed by linearization yields the dispersion relation

$$
\Omega^{2} \mu^{2}-\frac{4 \mu a_{1}^{2}}{\mu-V_{2}}-\left[\left(\frac{a_{1}^{2}}{\mu-V_{2}}+\frac{a_{2}^{2}}{\mu-V_{1}}\right) \frac{\mu}{\delta}-\delta\right]^{2}=0 .
$$

We expect that the baseband MI, corresponding to $\Omega \rightarrow 0$, is the prerequisite of RW formation [29]. Generally, MI requires a nonzero imaginary part of $\mu$. In the baseband limit (i.e., letting $\Omega=0$ ), Eq. (35) will reduce to a real-coefficient quartic equation $(B \neq 0)$ whose solution $\mu$ can be solved algebraically. It is easy to prove that as $V_{1}>V_{2}$ (the SE case), 

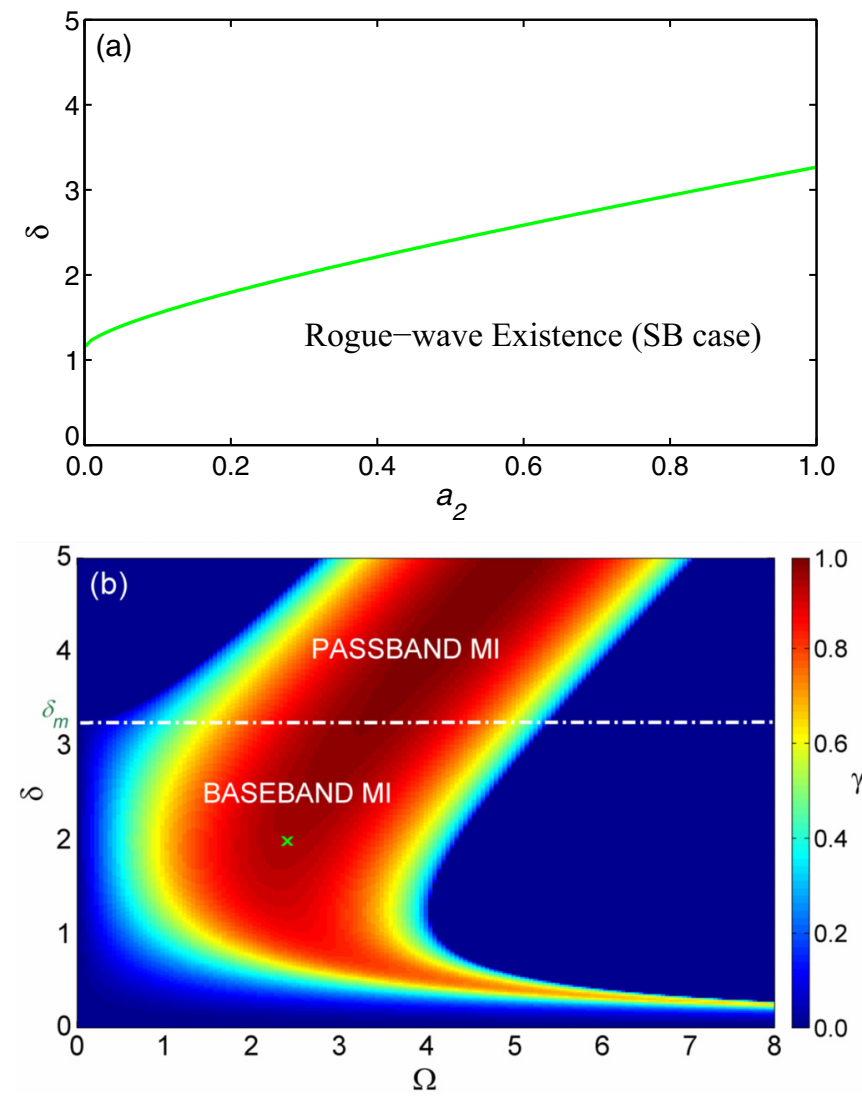

FIG. 8. (Color online) (a) Existence regime of the RWs in the plane $\left(a_{2}, \delta\right)$, plotted for $V_{1}=1, V_{2}=4$, and $a_{1}=2$. (b) Map of the MI gain in the $(\Omega, \delta)$ plane, divided into the baseband and passband parts by the dash-dotted line, calculated for the given parameters $V_{1}=1, V_{2}=4, a_{1}=2$, and $a_{2}=1$. The green cross in the map indicates the maximum growth rate for $\delta=2$, which corresponds to a modulation frequency of $\Omega_{\max }=2.41$.

Eq. (35) always permits a pair of complex conjugate roots in the whole regime of $\delta$, whereas for $V_{1}<V_{2}$ (the SB case), it only supports complex roots when the parameter condition (10) is fulfilled, suggesting that the baseband-MI conjecture is once again validated when contrasted with the analytical derivation. To give an illustration of the SB case, we show in Fig. 8(a) the existence regime of RWs in the parameter space $\left(a_{2}, \delta\right)$, by solving Eq. (35) with $\Omega=0, V_{1}=1, V_{2}=4$, and $a_{1}=2$. It is clear that as $a_{2}=1$, the maximum value of $\delta$ for the RW existence is around 3.26, agreeing well with the analytical value $\delta_{m}=4 \sqrt{2 / 3}$ shown in Fig. 1(a).

To gain more insight from the baseband-MI conjecture, we numerically solved the sextic equation (35), and plot in Fig. 8(b) the growth rate of the MI gain, defined by $\gamma=$ $\Omega \operatorname{Im}(\mu)$, versus the modulation frequency $\Omega$ and the frequency difference $\delta$. The other parameters are specified by $V_{1}=1$, $V_{2}=4, a_{1}=2$, and $a_{2}=1$. It is seen that the MI map consists of baseband and passband domains, but only the baseband one may lead to the generation of RWs.

\section{NUMERICAL SIMULATIONS}

Lastly, we performed extensive numerical simulations in order to study the evolution dynamics of TWRI RWs in either the degenerate or nondegenerate situation, based on the standard split-step Fourier method [19,40,43]. As in Ref. [19], our numerical code has reproduced the analytical solutions very well. Here we are primarily concerned with the stability of these TWRI RWs with respect to background broadband noise sources (e.g., quantum noise), an issue that may interest the general soliton community. In fact, recent work has demonstrated that the recurrent behavior (or FermiPasta-Ulam recurrence) of Akhmediev breather solutions of the scalar NLS equation may eventually break down in the presence of competing spontaneous noise-activated MI [49]. To this end, we perturbed the initial deterministic RW profiles by small amounts of white noise, and inspected whether the RWs generation is still observed in the presence of the competing spontaneous MI.

To be specific, we multiplied the real and imaginary parts of all the three field components $u_{n}$ at sufficient negative times (where the RW solution is almost imperceptible) by a factor $\left[1+\varepsilon r_{i}(x)\right](i=1, \ldots, 6)$, respectively, where $r_{i}$ are six uncorrelated random functions uniformly distributed in the

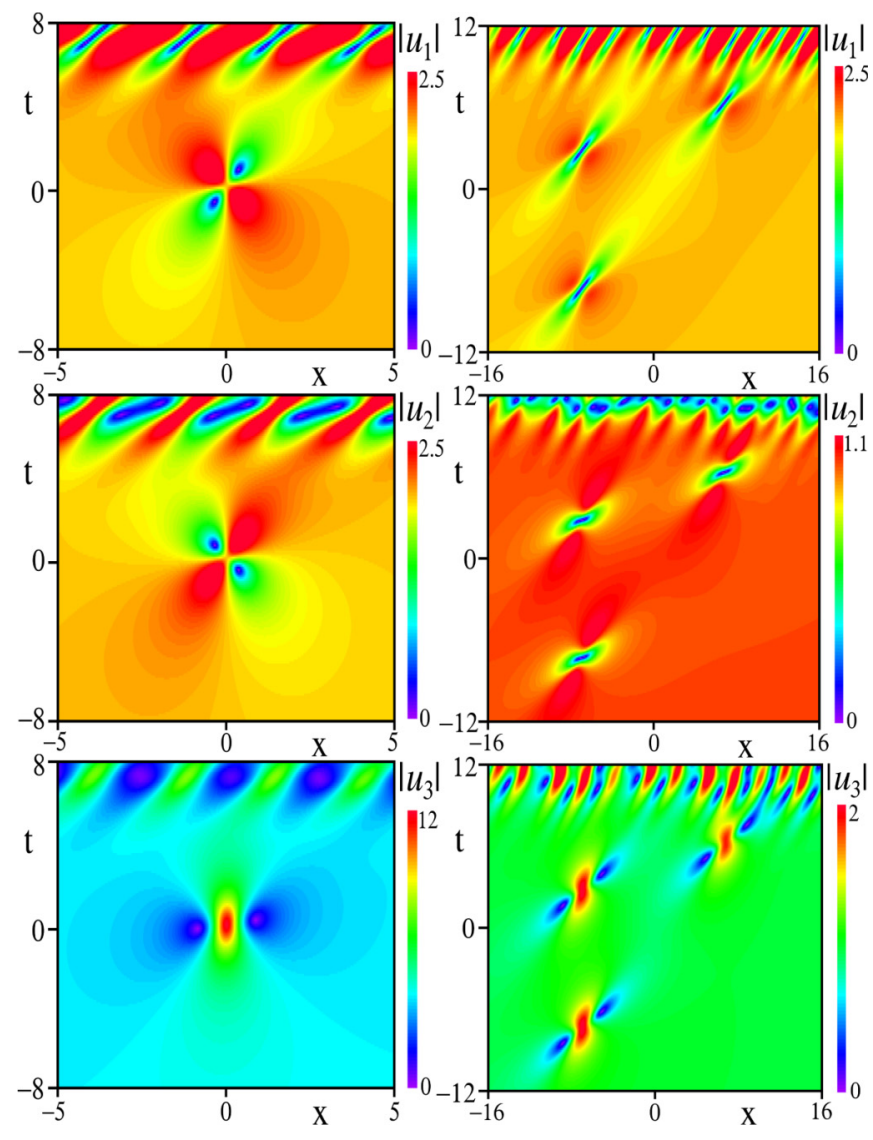

FIG. 9. (Color online) Simulation results of the RW states under initial white-noise perturbations. Left column: the IIB RWs occurring in the degenerate situation $\left(\varepsilon=10^{-6}\right)$; right column: the DDB RW triplets in the SB situation $\left(\varepsilon=10^{-7}\right)$. The other initial parameters for these two types of RW states are the same as in Figs. 4 and 5 (left), respectively. 
interval $[-1,1]$ and $\varepsilon$ is a small parameter defining the noise level. Shown in Fig. 9 are the numerical results corresponding to the IIB RWs in the degenerate situation (left column)
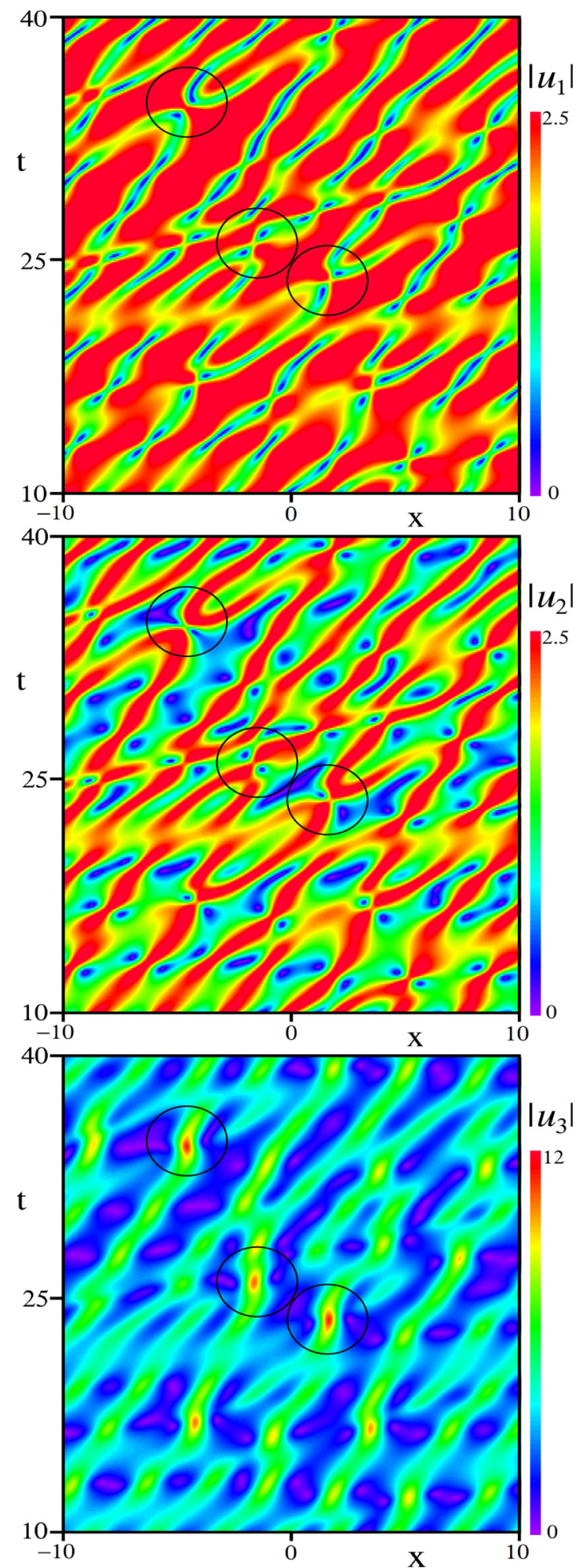

FIG. 10. (Color online) Numerical excitation of the RW states with a degenerate group velocity $V_{1}=V_{2}$, indicated by the circles, from a white-noise perturbation to the initial plane-wave solutions (2), under otherwise identical parameters as in Fig. 4. and the DDB triplets occurring in the SB situation (right column), perturbed by a noise intensity of $\varepsilon=10^{-6}$ and $10^{-7}$, respectively. Their corresponding unperturbed versions (i.e., the numerical results without white-noise perturbations) are the same as illustrated in Figs. 4 and 5 (left column). As seen, in the presence of a tiny perturbation, either the fundamental or the second-order RWs can still propagate very neatly for a rather long time, till eventually the spontaneous MI of the background fields grows up. Usually, when a larger noise intensity is adopted, noise-induced MI tends to interfere more strongly with the trailing edge of the RWs. Yet, in spite of the intrinsic instability of the background, the RWs themselves are typically robust enough to fully develop [19,40,41,43]. Indeed, the RW generation process may be thought of as induced MI, which is generally prevailing over spontaneous MI at least over relatively short propagation distances.

On the other side, one can also compare the numerical simulations with the MI analysis, if the same initial planewave parameters are used. We note that in Fig. 8(b), when $\delta=\delta_{a}=2$, the maximum gain (green cross) corresponds to a modulation frequency of 2.41. In the meantime, in Fig. 9 (right column) where we used the same initial parameters, it is shown that the period of the MI-induced waves is around $32 / 12$, corresponding to a modulation frequency of $3 \pi / 4 \simeq 2.36$, almost the same as revealed in the MI analysis. Obviously, this good consistency further confirms the soundness of our numerical results.

In order to see whether the deterministic RW solutions could indeed be excited in realistic random initial field conditions, we performed additional numerical simulations where we only added initial white noise on top of the plane-wave solutions (2) directly. That is, we considered the case of spontaneously induced MI with no initial deterministic seed. A typical example of our results is provided in Fig. 10, where we used identical parameters as in Fig. 9 (left column), except for the initial wave profiles which are now different. It is clearly seen that, after a propagation of 25 time units or so, the RWs in the degenerate situation, which manifest themselves by the IIB structures, could still be generated from a random wave field; see the wave patterns encircled by a black line. In fact, one can see many similar IIB structures in this portion of the sea of waves. We have ringed just a few of them for illustrative purposes.

\section{CONCLUSIONS}

In conclusion, in this work we presented a systematic study of the complex RW dynamics that originates either from the $\mathrm{SE}$ process in phase-matched weakly dispersive quadratic media or the SB process in transient stimulated scattering. The explicit RW solutions up to the second order to the TWRI equations are given, revealing that markedly different vector RW behaviors are allowed by the different resonant interaction processes. Specifically, in the SB situation, there may exist two or three RW components which can be dark, while in the SE situation, there are usually two or three RW components that would be bright, each holding true for both the fundamental and the triplet dynamics. Besides, within the framework of the energy exchange, the RWs in the SB situation exhibit a less drastic energy flow than in the SE situation. 
We also demonstrate the RW dynamics in the degenerate situation, where two interacting optical fields share the same group velocity. It is revealed that the sum of the intensities of this pair of optical wave components is spatiotemporally invariant, a property somehow linked to the related behavior of the sine-Gordon equation [47]. This dynamical property is completely different from what occurs in the pure SE or SB scenario, where this sum is not conserved.

The link between the existence of RW solutions and the presence of baseband MI is then reasserted, by an analysis of the MI gain map in the SB situation. We show once again that, although the MI consists of baseband and passband domains, only the baseband one is responsible for the generation of RWs [29]. In this regard, the baseband-MI theory could offer an alternative method for calculating the parametric conditions for the existence of RWs.

We finally performed extensive numerical simulations to confirm the possible manifestation of these vector RWs in the presence of background white-noise perturbations. We show that the RWs in TWRI systems can be robust enough to develop in spite of the onset of spontaneous MI. The numerical excitation of RW states from a random wave field was also demonstrated. In view of the importance and universality of the TWRI model in physics [1], we anticipate that our findings, either from analytical predictions or from numerical simulations, may provide a deeper understanding of RW phenomena occurring in resonant optical media [4,5] and laser-plasma interactions [13], as well as in fluid dynamics (e.g., capillary-gravity waves) [50].

\section{ACKNOWLEDGMENTS}

This work was supported by the National Natural Science Foundation of China (Grants No. 11174050 and No. 11474051) and by the Italian Ministry of University and Research (MIUR, Project No. 2012BFNWZ2). Ph.G. was supported by the Agence Nationale de la Recherche (Projects No. ANR-2010-BLANC-0417-01 and No. ANR-2012-BS040011). The work of J.M.S.C. was supported by MINECO under Contract No. TEC2012-37958-C02-02, by C.A.M. under Contract No. S2013/MIT-2790, and by the Volkswagen Foundation. M.C. acknowledges the support from the Agence Nationale de la Recherche (Project No. ANR-14-ACHN-0014 NoAWE). F.B. and S.W. are also with Istituto Nazionale di Ottica del Consiglio Nazionale delle Ricerche.
[1] D. J. Kaup, A. Reiman, and A. Bers, Rev. Mod. Phys. 51, 275 (1979).

[2] G. P. Agrawal, Nonlinear Fiber Optics, 5th ed. (Academic, New York, 2013), p. 370.

[3] A. Degasperis, M. Conforti, F. Baronio, S. Wabnitz, and S. Lombardo, Lett. Math. Phys. 96, 367 (2011).

[4] E. Ibragimov and A. Struthers, Opt. Lett. 21, 1582 (1996).

[5] M. Conforti, F. Baronio, A. Degasperis, and S. Wabnitz, Opt. Express 15, 12246 (2007).

[6] E. Picholle, C. Montes, C. Leycuras, O. Legrand, and J. Botineau, Phys. Rev. Lett. 66, 1454 (1991).

[7] A. Abdolvand, A. Nazarkin, A. V. Chugreev, C. F. Kaminski, and P. St. J. Russell, Phys. Rev. Lett. 103, 183902 (2009).

[8] F. Baronio, M. Andreana, M. Conforti, G. Manili, V. Couderc, C. De Angelis, and A. Barthélémy, Opt. Express 19, 13192 (2011).

[9] G. N. Burlak and N. Y. Kotsarenko, Sov. Tech. Phys. Lett. 10, 284 (1984); G. N. Burlak, N. Y. Kotsarenko, and Y. N. Taranenko, ibid. 14, 840 (1988).

[10] Y. N. Taranenko and L. G. Kazovsky, IEEE Photon. Technol. Lett. 4, 494 (1992); N. L. Taranenko, L. G. Kazovsky, and Y. N. Taranenko, J. Lightwave Technol. 12, 1101 (1994).

[11] A. Picozzi and M. Haelterman, Phys. Rev. Lett. 86, 2010 (2001); M. Conforti, F. Baronio, A. Degasperis, and S. Wabnitz, Phys. Rev. E 74, 065602(R) (2006).

[12] F. Baronio, M. Conforti, A. Degasperis, and S. Wabnitz, IEEE J. Quantum Electron. 44, 542 (2008).

[13] V. M. Malkin, G. Shvets, and N. J. Fisch, Phys. Rev. Lett. 82, 4448 (1999); Yu. A. Tsidulko, V. M. Malkin, and N. J. Fisch, ibid. 88, 235004 (2002).

[14] V. E. Zakharov and S. V. Manakov, JETP Lett. 18, 243 (1973); F. Baronio, M. Conforti, M. Andreana, V. Couderc, C. De Angelis, S. Wabnitz, A. Barthélémy, and A. Degasperis, Opt. Express 17, 13889 (2009).
[15] A. Buryak, P. Di Trapani, D. Skryabin, and S. Trillo, Phys. Rep. 370, 63 (2002).

[16] A. Degasperis, M. Conforti, F. Baronio, and S. Wabnitz, Phys. Rev. Lett. 97, 093901 (2006); F. Baronio, M. Conforti, C. De Angelis, A. Degasperis, M. Andreana, V. Couderc, and A. Barthélémy, ibid. 104, 113902 (2010).

[17] M. Conforti, F. Baronio, and A. Degasperis, Physica D 240, 1362 (2011).

[18] F. Baronio, M. Conforti, A. Degasperis, and S. Lombardo, Phys. Rev. Lett. 111, 114101 (2013); A. Degasperis and S. Lombardo, Phys. Rev. E 88, 052914 (2013).

[19] S. Chen, J. M. Soto-Crespo, and Ph. Grelu, Opt. Express 23, 349 (2015).

[20] C. Bonatto, M. Feyereisen, S. Barland, M. Giudici, C. Masoller, J. R. R. Leite, and J. R. Tredicce, Phys. Rev. Lett. 107, 053901 (2011); S. Birkholz, C. Brée, A. Demircan, and G. Steinmeyer, ibid. 114, 213901 (2015).

[21] D. R. Solli, C. Ropers, P. Koonath, and B. Jalali, Nature (London) 450, 1054 (2007).

[22] C. Lecaplain, Ph. Grelu, J. M. Soto-Crespo, and N. Akhmediev, Phys. Rev. Lett. 108, 233901 (2012); J. M. Soto-Crespo, Ph. Grelu, and N. Akhmediev, Phys. Rev. E 84, 016604 (2011); C. Lecaplain and Ph. Grelu, Phys. Rev. A 90, 013805 (2014).

[23] S. Birkholz, E. T. J. Nibbering, C. Brée, S. Skupin, A. Demircan, G. Genty, and G. Steinmeyer, Phys. Rev. Lett. 111, 243903 (2013).

[24] P. Walczak, S. Randoux, and P. Suret, Phys. Rev. Lett. 114, 143903 (2015).

[25] J. M. Dudley, F. Dias, M. Erkintalo, and G. Genty, Nat. Photon. 8, 755 (2014).

[26] N. Akhmediev, A. Ankiewicz, and M. Taki, Phys. Lett. A 373, 675 (2009); N. Akhmediev, A. Ankiewicz, and J. M. SotoCrespo, Phys. Rev. E 80, 026601 (2009). 
[27] C. Kharif, E. Pelinovsky, and A. Slunyaev, Rogue Waves in the Ocean (Springer, Berlin, 2009).

[28] M. Onorato, S. Residori, U. Bortolozzo, A. Montina, and F. T. Arecchi, Phys. Rep. 528, 47 (2013).

[29] F. Baronio, M. Conforti, A. Degasperis, S. Lombardo, M. Onorato, and S. Wabnitz, Phys. Rev. Lett. 113, 034101 (2014); F. Baronio, S. Chen, Ph. Grelu, S. Wabnitz, and M. Conforti, Phys. Rev. A 91, 033804 (2015).

[30] A. Chabchoub, N. P. Hoffmann, and N. Akhmediev, Phys. Rev. Lett. 106, 204502 (2011).

[31] A. Chabchoub, N. Hoffmann, M. Onorato, and N. Akhmediev, Phys. Rev. X 2, 011015 (2012).

[32] M. Shats, H. Punzmann, and H. Xia, Phys. Rev. Lett. 104, 104503 (2010); H. Xia, T. Maimbourg, H. Punzmann, and M. Shats, ibid. 109, 114502 (2012).

[33] H. Bailung, S. K. Sharma, and Y. Nakamura, Phys. Rev. Lett. 107, 255005 (2011).

[34] B. Kibler, J. Fatome, C. Finot, G. Millot, F. Dias, G. Genty, N. Akhmediev, and J. M. Dudley, Nat. Phys. 6, 790 (2010).

[35] Yu. V. Bludov, V. V. Konotop, and N. Akhmediev, Phys. Rev. A 80, 033610 (2009).

[36] D. H. Peregrine, J. Aust. Math. Soc. Ser. B, Appl. Math. 25, 16 (1983).

[37] A. Ankiewicz, J. M. Soto-Crespo, and N. Akhmediev, Phys. Rev. E 81, 046602 (2010); A. Ankiewicz, J. M. Soto-Crespo, M. A. Chowdhury, and N. Akhmediev, J. Opt. Soc. Am. B 30, 87 (2013).
[38] U. Bandelow and N. Akhmediev, Phys. Rev. E 86, 026606 (2012); S. Chen, ibid. 88, 023202 (2013); J. M. Soto-Crespo, N. Devine, N. P. Hoffmann, and N. Akhmediev, ibid. 90, 032902 (2014).

[39] F. Baronio, A. Degasperis, M. Conforti, and S. Wabnitz, Phys. Rev. Lett. 109, 044102 (2012).

[40] S. Chen, Ph. Grelu, and J. M. Soto-Crespo, Phys. Rev. E 89, 011201(R) (2014).

[41] S. Chen, J. M. Soto-Crespo, and Ph. Grelu, Phys. Rev. E 90, 033203 (2014); S. Chen, Phys. Lett. A 378, 2851 (2014).

[42] L. Ling, B. Guo, and L.-C. Zhao, Phys. Rev. E 89, 041201(R) (2014); S. Chen and D. Mihalache, J. Phys. A: Math. Theor. 48, 215202 (2015).

[43] S. Chen, J. M. Soto-Crespo, and Ph. Grelu, Opt. Express 22, 27632 (2014).

[44] A. Ankiewicz, D. J. Kedziora, and N. Akhmediev, Phys. Lett. A 375, 2782 (2011); A. Chabchoub and N. Akhmediev, ibid. 377, 2590 (2013).

[45] Y. Ohta and J. Yang, Proc. R. Soc. A 468, 1716 (2012); J. S. He, H. R. Zhang, L. H. Wang, K. Porsezian, and A. S. Fokas, Phys. Rev. E 87, 052914 (2013).

[46] D. J. Kedziora, A. Ankiewicz, and N. Akhmediev, Phys. Rev. E 84, 056611 (2011); 88, 013207 (2013).

[47] M. Conforti and F. Baronio, Eur. Phys. J. D 64, 115 (2011).

[48] S. Chen, Y. Liu, and A. Mysyrowicz, Phys. Rev. A 81, 061806(R) (2010).

[49] S. Wabnitz and B. Wetzel, Phys. Lett. A 378, 2750 (2014).

[50] A. D. D. Craik, Wave Interactions and Fluid Flows (Cambridge University Press, Cambridge, UK, 1988). 